\title{
Efficient Human Hand Kinematics for Manipulation Tasks
}

\author{
Salvador Cobos, Manuel Ferre, M.A. Sanchéz Urán, Javier Ortego and Cesar Peña
}

\begin{abstract}
This work is focused on obtaining efficient human hand models that are suitable for manipulation tasks. A 24 DoF kinematic model of the human hand is defined to realistic movements. This model is based on the human skeleton. Dynamic and Static constraints have been included in order to improve the movement realism. Two simplified hand models with 9 and 6 DoF have been developed according to the constraints predefined. These simplified models involve some errors in reconstructing the hand posture. These errors are calculated with respect to the $24 \mathrm{DoF}$ model and evaluated according to the hand gestures. Finally, some criteria are defined to select the hand description best suited to the features of the manipulation task.
\end{abstract}

\section{INTRODUCTION}

Different models of the human hand currently exist. [1] describes a hand model with 26 DoF, [2] describes a hand model with 23 Degrees of Freedom (DoF), [3], [4] proposes a hand model with $20 \mathrm{DoF}$ plus $2 \mathrm{DoF}$ for the wrist and $2 \mathrm{DoF}$ for the arm, and [5] proposes a hand model with 26 DoF. A human hand model of 24 DoFs is described in the next section. This model represents a balance between complexity and realism. It is important to indicate that more or less DoF can result in increased complexity or a decreased range of movement. Therefore, a suitable kinematic model is needed in order to conserve all the kinematic information for object manipulation. A significant simplification is done in the original hand description. Therefore, the most relevant finger relations and constraints have been analyzed in order to define new simplified hand models. These constraints have been obtained from literature and experiments that demonstrate the strong finger coupling in manipulation. As result, two new simplified hand model descriptions with 9 and 6 DoFs have been defined. These models represent a significant reduction in the processed information. Finally, some experiments have been carried out in order to evaluate the position errors that involve these simplified hand model descriptions. According to the experiments, position errors

\footnotetext{
Manuscript received February 22, 2008. This work is supported by the European commission under the IMMERSENCE integrated project of the sixth frame program (IST-4-027141-IP).

S. Cobos is with The Group of intellingent Machines of Universidad Politécnica de Madrid, C/José Gutiérrez Abascal, 228006 , Madrid, Spain. Phone:(+34) 913363195; fax:(+34) 913363010. e-mail: cobosalvador@etsii.upm.es

M. Ferre is with The Group of intellingent Machines of Universidad Politécnica de Madrid, e-mail: mferre@etsii.upm.es

M. A. Sanchéz-Urán is with The Group of intellingent Machines of Universidad Politécnica de Madrid, e-mail miguelangel.sanchezuran@upm.es

J. Ortego is with The Group of intellingent Machines of Universidad

Politécnica de Madrid, e-mail: jortego@etsii.upm.es

C. Peña is with The Group of intellingent Machines of Universidad Politécnica de Madrid, e-mail: caugusto@etsii.upm.es
}

are around $5 \%$ for the 9 DoFs hand description and $10 \%$ for the 6 DoFs. A Cyberglove $\mathbb{R}$ [6] has been used for experiments carried out in this paper. Intra-finger and interfinger constraints have been checked with the information provided by the glove. Finally, Cyberglove has been used for evaluating the error of the simplified hand description versus the full 24 DoFs hand model. These experiments compare finger trajectories for circular and prismatic grasping by using 24, 9 and 6 DoFs. This paper is organized as follows: section 2 describes the 24 DoFs kinematic model of the hand. Section 3 shows the main constraints of the finger movements. Intra-finger constraints for circular and prismatic grasping are obtained. Section 4 describes the 9 and 6 DoF simplified hand descriptions and their corresponding position errors for grasping. Finally, conclusions are presented in section 5 .

\section{Kinematic Human Hand Model}

The hand model used for this work is based on the human skeleton. The kinematic model is comprised of 19 links that imitate the corresponding human bones, and 24 degrees of freedom (DoF) that represent the joints. Two kinematic configurations are considered in this model, one for the thumb and other for the rest of the fingers. Therefore, same kinematic configuration is used for index, middle, ring and little fingers which are defined by 4 links and 5 DoFs. In these fingers, metacarpophalangeal joint (MCP) is modeled by a $2 \mathrm{DoF}$ universal joint whereas carpometacarpal (CMC), proximal interphalangeal (PIP) and distal interphalangeal (DIP) have 1 DoF. Thumb finger is modeled by 3 links and $4 \mathrm{DoF}$. The trapeziometacarpal (TMC) thumb joint is also defined by a 2 DoF universal joint whereas metacarpophalangeal (MCP) and interphalangeal (IP) joints are modeled by 1 DoF. Figure 1 shows details of this kinematics models. Main points of this model are the use of four DoF for the thumb finger modeling, the inclusion of the CMC joint and the movement concatenation in the MCP joint. The MCP abduction/adduction turn is defined first than the MCP flexion in order to better simulate finger displacements. The CMC joint allows simulating the palm arc; it represents the deformation in the palm when the hand is grasping a ball or similar objects. The above points contribute to define high realistic hand movements and gestures.

\section{A. Direct Kinematics}

Direct kinematic equations are used to obtain the finger tip position and orientation according to the joint angles. These direct kinematics models are obtained for the two cases defined (thumb and rest of fingers). Model equations are 

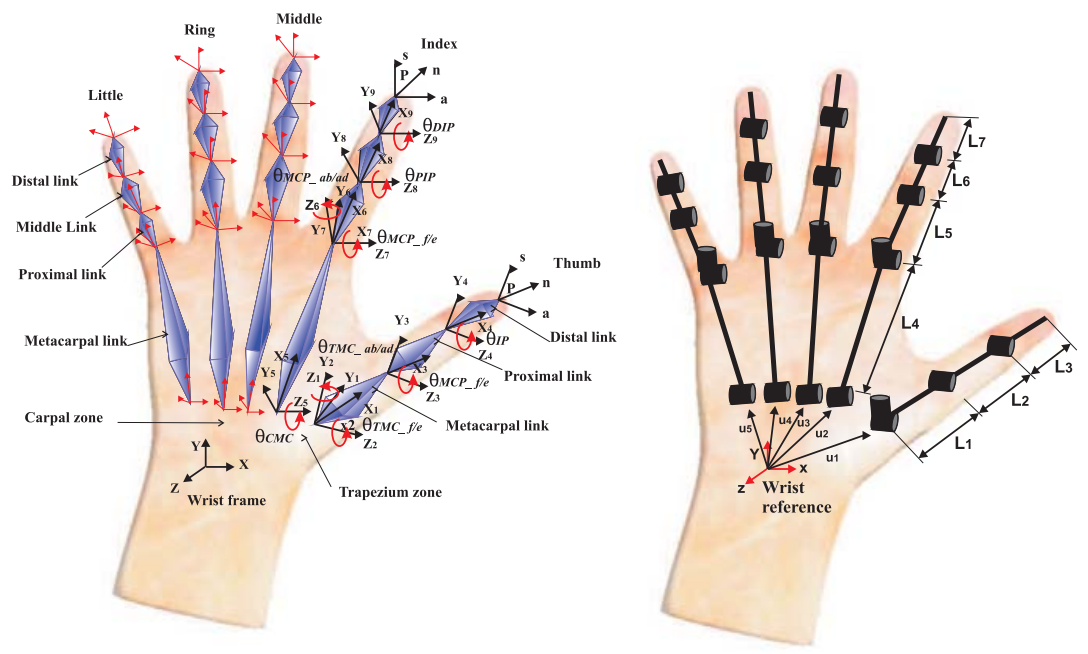

Fig. 1. Kinematic configuration of the human hand.Thumb is defined by 3 links and 4 degrees of freedom whereas index, middle, ring and little are defined by 4 links and 5 DoFs.

calculated by means of the Denavit-Hartenberg (D-H) parameters [7]. This convention is commonly used for mechanism and robotics modeling. Human anatomical terminology has been used to describe the hand model. Direct kinematic equations are required when is simulated a virtual grasping. That is, a user is wearing a glove that provides the human joints and direct kinematic equations provide what fingertip positions and orientations are. This information will be used to calculate object contact points, grasping routes, etc.

1) Direct kinematics of the index, middle, ring and little fingers: D-H parameters have been used for defining index, middle, ring and little finger configurations. The four fingers have four bones: metacarpal, proximal, middle and distal. These bones represent the length of each link of the serial kinematic chain. The corresponding joints to these fingers are: carpometacarpal (CMC) joint, metacarpophalangeal (MCP) joint, proximal interphalangeal (PIP) joint and distal interphalangeal (DIP) joint. The MCP joint has 2 degrees of freedom that define the adduction/abduction and flexion/extension movements. The CMC, PIP and DIP joints are of the flexion/extension type with respect to the sagittal plane of the hand. It is advisable to highlight that a vector

\begin{tabular}{|c|c|c|c|c|}
\hline Joint & $\theta_{i}$ & $d_{i}$ & $a_{i}$ & $\alpha_{i}$ \\
\hline 1 & $\theta_{\text {CMC }}$ & 0 & $L_{4}$ & $\pi / 2$ \\
2 & $\theta_{\text {MCPab/ad }}$ & 0 & 0 & $-\pi / 2$ \\
3 & $\theta_{\text {MCPf/e }}$ & 0 & $L_{5}$ & 0 \\
4 & $\theta_{P I P}$ & 0 & $L_{6}$ & 0 \\
5 & $\theta_{\text {DIP }}$ & 0 & $L_{7}$ & 0 \\
\hline
\end{tabular}

TABLE I

D-H PARAMETERS FOR THE FIRST MODEL

is used for defining the reference frame origin of each finger with regard to the wrist reference, such as in Figure 1 (right hand). Table I shows the D-H parameters for index, middle, ring and little fingers. Equation 1 shows the direct kinematics of index to little fingers.

- $p_{i}$ represents a matrix that containts position and orientation of the finger tip,

- ${ }_{-1}^{0} T\left(u_{i}\right)$ represents the distance between wrist and the finger reference frame

- ${ }_{5}^{0} T\left(\theta_{j}\right) i$ is a matrix that containts the geometrical transformation between the i-finger reference frame and its corresponding finger tip. This matrix is composed by the concatenation of more simple matrixes that represents the contribution of each finger joint $\left(\theta_{C M C}, \theta_{M C P a d / a b}\right.$, $\theta_{M C P f / e}, \theta_{P I P}$ and $\left.\theta_{D I P}\right)$.

$$
\begin{aligned}
p_{i}={ }_{-1}^{0} T\left(u_{i}\right){ }_{5}^{0} T\left(\theta_{j}\right) i= & { }_{-1}^{0} T\left(u_{i}\right){ }_{1}^{0} T\left(\theta_{C M C}\right) i T\left(\theta_{M C P a b / a d}\right) i \\
& T\left(\theta_{M C P f / e}\right) i T\left(\theta_{P I P}\right) i T\left(\theta_{D I P}\right) i
\end{aligned}
$$

$i=$ index, middle, ring and little

$j=\theta_{C M C}, \theta_{M C P a b / a d}, \theta_{M C P f / e}, \theta_{P I P}$ and $\theta_{D I P}$

2) Direct kinematics of the thumb: The thumb has been modeled by four principal bones: trapezium, metacarpal, proximal and distal. The joints corresponding to these fingers are: trapeziometacarpal (TMC), metacarpophalangeal (MCP) and interphalangeal (IP) joints. The TMC joint has $2 \mathrm{DoF}$ in Flexion/Extension and Adduction/Abduction. Table II shows the D-H parameters for the thumb model. Equation 2 represents the direct kinematics for thumb finger. Terms are similar to the equation 1 .

$$
\begin{array}{r}
p_{\text {Thumb }}={ }_{-1}^{0} T\left(u_{\text {Thumb }}\right){ }_{4}^{0} T\left(\theta_{j}\right){ }_{\text {Thumb }}= \\
{ }_{-1}^{0} T\left(u_{\text {Thumb }}\right){ }_{1}^{0} T\left(\theta_{\text {TMCab } / a d}\right){ }_{2}^{1} T\left(\theta_{\text {TMCf } / e}\right){ }_{3}^{2} T\left(\theta_{M C P}\right){ }_{4}^{3} T\left(\theta_{I P}\right)
\end{array}
$$

\section{B. Inverse Kinematics}

The model of the two finger types described in the direct kinematics section can generate a combination of movement 


\begin{tabular}{|c|c|c|c|c|}
\hline Joint & $\theta_{i}$ & $d_{i}$ & $a_{i}$ & $\alpha_{i}$ \\
\hline 1 & $\theta_{T M C a b / a d}$ & 0 & 0 & $\pi / 2$ \\
2 & $\theta_{T M C f / e}$ & 0 & $L_{1}$ & 0 \\
3 & $\theta_{\text {MCPfle }}$ & 0 & $L_{2}$ & 0 \\
4 & $\theta_{I P}$ & 0 & $L_{3}$ & 0 \\
\hline
\end{tabular}

TABLE II

D-H PARAMETERS FOR THE THUMB

with flexions, extensions, abductions, adductions and redundant cases. Solution of the inverse kinematics can be derived from geometric methods [8], such as the relation of triangles. The hand can reproduce positive or negative movements with regard to a reference line for some joints. The movements of the fingers that can be in two different quadrants are: flexion/extension and abduction/adduction. The inverse kinematics is solved in all these cases of movement. In addition, the kinematic behavior of the kinematic chain depends on the MCP abduction/adduction for the first finger model, because this joint can cause situations such as redundancy (Figure 2) if the value of MCP abduction/adduction $=0$. Also, this joint can produce a more complex situation if the adduction is high. The most stable behavior of the kinematic chain is when there are only abductions. Kinematically, the

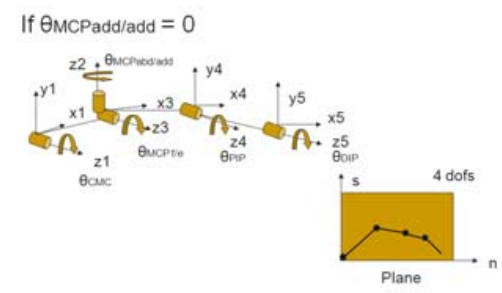

Fig. 2. Redundant case of the first finger model

model of the human hand is a redundant case. Therefore several solutions exist. In this type of situation, the inverse kinematics can be solved by means of iterative methods such as the Newton - Raphson method that uses the Jacobean matrix [9] and [10]. To solve this redundant case correctly, constrains have been implemented to solve with a convergent solution.

1) Inverse kinematics of the first finger model.: To solve the inverse problem for each finger, the first step to do consist of measuring the orientation and position end of the fingertip. With this, it is possible to obtain a homogeneous matrix [n $\mathrm{s}$ a P]. Algebraically, for the first degree of freedom of the $\mathrm{CMC}$ joint is obtained:

$$
\theta_{C M C}=\operatorname{atan}\left[\frac{a_{2 y}}{a_{2 x}}\right]
$$

In the same way, finding the value of the CMC joint became obtained of algebraic way, the MCP abduction/adduction joint as

$$
\theta_{M C P(a b / a d)}=\operatorname{atan}\left[\frac{a_{2} x}{a_{2 z} \cos \theta_{C M C}}\right]
$$

There exists dependency among the following joints: flexions for MCP (f/e), PIP and DIP. They are solved by means

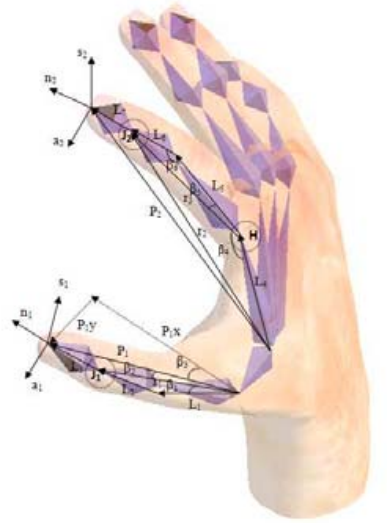

Fig. 3. Inverse kinematics

of geometric method. From P2 information vectorially is possible to obtain a point $\mathrm{J} 2$ with the following expression:

$$
J_{2}=P_{2}-\left[L_{7} * n_{2}\right]
$$

Another vector $\mathrm{H}$ is calculated with the CMC information and length $\mathrm{L} 4$ of the link metacarpal such as:

$$
\begin{gathered}
H_{x}=L_{4} \cos \theta_{C M C} ; \quad H_{y}=L_{4} \sin \theta_{C M C} ; \quad H_{z}=0 \\
\hat{H}=\left[\begin{array}{lll}
H_{x} & H_{y} & H_{z}
\end{array}\right]^{T}
\end{gathered}
$$

With information $\mathrm{J} 2$ and $\mathrm{H}$ is obtained the vectors $\mathrm{u}, \mathrm{r} 2$ and $\mathrm{r} 3$ as is shows in figure 3 .

$$
\hat{u}=\hat{J}_{2}-\hat{H} ; \hat{r_{2}}=\operatorname{norm}\left(\hat{J}_{2}\right) ; \hat{r_{3}}=\operatorname{norm}(\hat{u})
$$

$$
\beta_{4}=\operatorname{acos}\left[\frac{L_{4}^{2}+r_{3}^{2}-r_{2}^{2}}{2 L_{4} r_{3}}\right] ; \beta_{5}=\operatorname{acos}\left[\frac{L_{5}^{2}+r_{3}^{2}-L_{6}^{2}}{2 L_{5} r_{3}}\right]
$$

MCP flexion/extension is obtained as:

$$
\theta_{M C P(f / e)}=\pi-\beta_{5}-\beta_{4}
$$

The extension $\&$ hyperextension for MCP joint is obtained as:

$$
\theta_{M C P(h y p)}=\beta_{4}-\pi-\beta_{5}
$$

PIP joint is obtained as:

$$
\begin{gathered}
\beta_{6}=\operatorname{acos}\left[\frac{L_{6}^{2}+L_{5}^{2}-r_{3}^{2}}{2 L_{6} L_{5}}\right] \\
\theta_{P I P}=\pi-\beta_{6}
\end{gathered}
$$

Finally, DIP joint is obtained by algebraic method.

$$
\begin{aligned}
k_{1} & =\left(c_{2} c_{4} c_{3} c_{1}-c_{4} s_{1} s_{3}-c_{2} s_{4} c_{1} s_{3}-s_{4} c_{3} s_{1}\right) n_{x} \\
& +\left(c_{2} c_{4} c_{3} s_{1}+c_{4} c_{1} s_{3}-c_{2} s_{4} s_{1} s_{3}+s_{4} c_{3} c_{1}\right) n y \\
& +\left(\left(s_{4} s_{3}-c_{4} c_{3}\right) s_{2}\right) n z \\
k_{2} & =\left(-s_{4} c_{2} c_{1} c_{3}+s_{4} s_{1} s_{3}-c 4_{c} 1 c_{2} s_{3}-c_{4} c_{3} s_{1}\right) n_{x} \\
& +\left(-s_{4} c_{2} s_{1} c_{3}-s_{4} c_{1} s_{3}-c_{4} s_{1} c_{2} s_{3}+c_{4} c_{3} c_{1}\right) n_{y} \\
& +\left(\left(c_{4} s_{3}+s_{4} c_{3}\right) s_{2}\right) n z \\
\theta_{D I P} & =\operatorname{atan} 2\left[k_{2}, \quad k_{1}\right]
\end{aligned}
$$


2) Inverse kinematics of the thumb: For the thumb, inverse kinematics is obtained by means of algebraic method such as:

$$
\begin{gathered}
\theta_{T M C(a b / a d)}=\operatorname{atan}\left[\frac{P_{1 y}}{P_{1 x}}\right] \\
J_{1}=P_{1}-\left[L_{3} n_{1}\right] ; \quad \hat{r}_{1}=\operatorname{norm}\left(\hat{J}_{1}\right) \\
x_{1}=\frac{r_{1}-L_{1}^{2}-L_{2}^{2}}{2 L_{1} L_{2}} ; \quad x_{2}=\sqrt{1-x_{1}^{2}} \\
\theta_{M C P}=\operatorname{atan} 2\left[x_{2}, x_{1}\right] \\
x_{3}=\left(L_{1}+\left(L_{2} x_{1}\right)\right) \\
x_{5 y}=\frac{J_{1 z} x_{4}+J_{x y} x_{3}}{x_{3}^{2}+x_{4}^{2}} ; \quad x_{6}=\frac{J_{1 z} x_{3}-J_{x y} x_{4}}{x_{3}^{2}+x_{4}^{2}} \\
\theta_{T M C(f / e)}=\operatorname{atan} 2\left[x_{6}, x_{5}\right] \\
\theta=\operatorname{atan2}\left[n_{1 z}, \sqrt{n_{1 x}^{2}+n_{1 y}^{2}}\right] ; \quad \theta_{I P}=\theta-\theta_{T M C f / e}-\theta_{M C P}
\end{gathered}
$$

\section{MAIN CONSTRAINTS OF FINGER MOVEMENTS}

Joint finger movements are limited to a specific range because of static constraints, intra-finger constraints and inter-finger constraints. Intra-finger and inter-finger constraints are often called dynamic constraints, and these are the ones responsible for producing natural movements both statically and dynamically. However, this range of movement is somewhat ambiguous because the range depends on various factors involving human hand biomechanics. Main static constraints have been collected from several works [11], [12]. Finger movements are classified as active and passive. The active movements are actuated by muscles and tendons that permit a range of voluntary movements. The passive movements are a range of movements that are not actuated. Table III shows static constraints. In this paper, new constraints have been defined in the inter-finger and intrafinger categories, so as to obtain more realistic movements in the hand model. Intra-finger constraints have been developed to reproduce movements of finger trajectories such as circular and prismatic grasps [13]. Inter-finger constraints have been verified in experiments carried out with a Cyberglove [14] to obtain the dependency of tendons mainly among the middle, ring and little fingers.

\section{A. Inter-finger constraints}

This type of constraint refers to some dependency among fingers while they are in motion [15]. The inter-finger constraints were obtained by using the hand model and Cyberglove. These types of constraints are coupled movements among the index, middle, ring and little fingers. The relationship among angles with the middle, ring and little fingers has been measured to represent real movements of the hand model. The Cases of involuntary movements have been defined in [16]. Middle, ring and little fingers share common flexor tendons. It implies involuntary movements due to this strong coupling.

\begin{tabular}{|c|c|c|c|}
\hline Finger & Flexion & Extension & $\begin{array}{c}\text { abduction/ } \\
\text { adduction }\end{array}$ \\
\hline Thumb & & & \\
\hline Scapahoid-Trapezium (STM) & $0^{\circ}$ & $0^{\circ}$ & $0^{\circ}$ \\
\hline Trapeziometacarpal (TMC) & $50^{\circ}-90^{\circ}$ & $15^{\circ}$ & $45^{\circ}-60^{\circ}$ \\
\hline Metacarpophalangeal (MCP) & $75^{\circ}-80^{\circ}$ & $0^{\circ}$ & $0^{\circ}$ \\
\hline Interphalangeal (IP) & $75^{\circ}-80^{\circ}$ & $5^{\circ}-10^{\circ}$ & $0^{\circ}$ \\
\hline Index & & & \\
\hline Carpometacarpal (CMC) & $5^{\circ}$ & $0^{\circ}$ & $0^{\circ}$ \\
\hline Metacarpophalangeal (MCP) & $90^{\circ}$ & $30^{\circ}-40^{\circ}$ & $60^{\circ}$ \\
\hline Proximal interphalangeal (PIP) & $110^{\circ}$ & $0^{\circ}$ & $0^{\circ}$ \\
\hline Distal interphalangeal (DIP) & $80^{\circ}-90^{\circ}$ & $5^{\circ}$ & $0^{\circ}$ \\
\hline Middle & & & \\
\hline Carpometacarpal (CMC) & $5^{\circ}$ & $0^{\circ}$ & $0^{\circ}$ \\
\hline Metacarpophalangeal (MCP) & $90^{\circ}$ & $30^{\circ}-40^{\circ}$ & $45^{\circ}$ \\
\hline Proximal interphalangeal (PIP) & $110^{\circ}$ & $0^{\circ}$ & $0^{\circ}$ \\
\hline Distal interphalangeal (DIP) & $80^{\circ}-90^{\circ}$ & $5^{\circ}$ & $0^{\circ}$ \\
\hline Ring & & & \\
\hline Carpometacarpal (CMC) & $10^{\circ}$ & $0^{\circ}$ & $0^{\circ}$ \\
\hline Metacarpophalangeal (MCP) & $90^{\circ}$ & $30^{\circ}-40^{\circ}$ & $45^{\circ}$ \\
\hline Proximal interphalangeal (PIP) & $120^{\circ}$ & $0^{\circ}$ & $0^{\circ}$ \\
\hline Distal interphalangeal (DIP) & $80^{\circ}-90^{\circ}$ & $5^{\circ}$ & $0^{\circ}$ \\
\hline Little & & & \\
\hline Carpometacarpal (CMC) & $15^{\circ}$ & $0^{\circ}$ & $0^{\circ}$ \\
\hline Metacarpophalangeal (MCP) & $90^{\circ}$ & $30^{\circ}-40^{\circ}$ & $50^{\circ}$ \\
\hline Proximal interphalangeal (PIP) & $135^{\circ}$ & $0^{\circ}$ & $0^{\circ}$ \\
\hline Distal interphalangeal (DIP) & $90^{\circ}$ & $5^{\circ}$ & $0^{\circ}$ \\
\hline
\end{tabular}

TABLE III

ACTIVE/PASSIVE MOVEMENTS

1) Simplified hand description for circular grasping: Circular grasping implies a strong relation among finger joints. These relations are summarized in the Table IV. The most accepted intra-finger constraint is: $\theta_{D I P}=\frac{2}{3} \theta_{P I P}$. It has been analyzed by several researchers, such as [17] and has been efficiently checked in our experiments. According to table IV a significant reduction can be done in the number of DoF used for defining the grasping gesture. 9 DoFs $\left(\theta_{T M C(a b / a d)}, \theta_{I P-T}, \theta_{M C P(a b / a d)-I}, \theta_{D I P-I}, \theta_{D I P-M}, \theta_{D I P-R}\right.$, $\left.\theta_{C M C-L}, \theta_{M C P(a b / a d)-L}, \theta_{D I P-L}\right)$ have been considered for defining a circular grasping gesture. The rest of the 24 DoFs defined in section 2 are obtained according to the table IV. Main conclusions of this table are that thumb is defined by 2 DoFs, index by 2 DoFs, middle by 1 DoF, ring by 1 DoF and little by 3 DoFs. The ring is calculated by the little and middle joints. Therefore, the thumb, the index and the little are the most important fingers when defining circular gestures.

2) Simplified hand description for prismatic grasping : Prismatic grasping represents a less constraint among finger that circular grasping. In this cases $9 \operatorname{DoFs}\left(\theta_{T M C(a b / a d)-T}\right.$, $\theta_{I P-T}, \theta_{M C P(a b / a d)-I}, \theta_{D I P-I}, \theta_{D I P-M}, \theta_{D I P-R}, \theta_{C M C-L}$, $\left.\theta_{M C P(a b / a d)-L}, \theta_{D I P-L}\right)$ are used for defining a prismatic grasping gesture. The rest of the $24 \mathrm{DoFs}$ defined in section 2 are obtained according to the table V. Main conclusions of this table are that thumb is defined by $2 \mathrm{DoFs}$, index by 2 DoFs, middle by 1 DoF, ring by 1 DoF and little by 3 DoFs. 


\begin{tabular}{|c|c|c|c|c|}
\hline Thumb & Index & Middle & Ring & Little \\
\hline$\theta_{T M C a b / a d}$ & $\theta_{C M C-I}=$ & $\theta_{C M C-M}=$ & $\theta_{C M C-R}=$ & $\theta_{C M C-L}$ \\
& $\theta_{C M C-M}$ & $\frac{1}{2} \theta_{C M C-R}$ & $\frac{2}{3} \theta_{C M C-L}$ & \\
\hline$\theta_{T M C f / e}=$ & $\theta_{M C p a b / a d-I}$ & $\theta_{M C P a b / a d-M}=$ & $\theta_{M C P a b / a d-R}=$ & $\theta_{M C P a b / a d-L}$ \\
$\frac{11}{10} \theta_{M C P}$ & & $\frac{1}{5} \theta_{M C P a b / a d-I}$ & $\frac{1}{2} \theta_{M C P a b / a d-L}$ & \\
\hline$\theta_{M C P}=$ & $\theta_{M C P f / e-I}=$ & $\theta_{M C P f / e-M}=$ & $\theta_{M C P f / e-R}=$ & $\theta_{M C P f / e-L}=$ \\
$\frac{4}{5} \theta_{I P}$ & $\frac{4}{3} \theta_{P I P-I}$ & $\frac{4}{3} \theta_{P I P-M}$ & $\frac{4}{3} \theta_{P I P-R}$ & $\frac{4}{3} \theta_{P I P-L}$ \\
\hline$\theta_{I P}$ & $\theta_{P I P-I}=$ & $\theta_{P I P-M}=$ & $\theta_{P I P-R}=$ & $\theta_{P I P-L}=$ \\
& $\frac{3}{2} \theta_{D I P-I}$ & $\frac{3}{2} \theta_{D I P-M}$ & $\frac{3}{2} \theta_{D I P-R}$ & $\frac{3}{2} \theta_{D I P-L}$ \\
\hline & $\theta_{D I P-I}$ & $\theta_{D I P-M}$ & $\theta_{D I P-R}$ & $\theta_{D I P-L}$ \\
\hline
\end{tabular}

TABLE IV

INTRA-FINGER CONSTRAINTS FOR CIRCULAR GRASPING

\begin{tabular}{|c|c|c|c|c|}
\hline Thumb & Index & Middle & Ring & Little \\
\hline$\theta_{T M C a b / a d}$ & $\theta_{C M C-I}=$ & $\theta_{C M C-M}=$ & $\theta_{C M C-R}=$ & $\theta_{C M C-L}$ \\
& $\theta_{C M C-M}$ & $\frac{1}{2} \theta_{C M C-R}$ & $\frac{2}{3} \theta_{C M C-L}$ & \\
\hline$\theta_{T M C f / e}=$ & $\theta_{M C p a b / a d-I}$ & $\theta_{M C P a b / a d-M}=$ & $\theta_{M C P a b / a d-R}=$ & $\theta_{M C P a b / a d-L}$ \\
$\frac{10}{11} \theta_{M C P}$ & & $\frac{1}{5} \theta_{M C P a b / a d-I}$ & $\frac{1}{2} \theta_{M C P a b / a d-L}$ & \\
\hline$\theta_{M C P}=$ & $\theta_{M C P f / e-I}=$ & $\theta_{M C P f / e-M}=$ & $\theta_{M C P f / e-R}=$ & $\theta_{M C P f / e-L}=$ \\
$\frac{6}{5} \theta_{I P}$ & $\frac{3}{2} \theta_{P I P-I}$ & $\frac{3}{2} \theta_{P I P-M}$ & $\frac{3}{2} \theta_{P I P-R}$ & $\frac{3}{2} \theta_{P I P-L}$ \\
\hline$\theta_{I P}$ & $\theta_{P I P-I}=$ & $\theta_{P I P-M}=$ & $\theta_{P I P-R}=$ & $\theta_{P I P-L}=$ \\
& $2 \theta_{D I P-I}$ & $2 \theta_{D I P-M}$ & $2 \theta_{D I P-R}$ & $2 \theta_{D I P-L}$ \\
\hline & $\theta_{D I P-I}$ & $\theta_{D I P-M}$ & $\theta_{D I P-R}$ & $\theta_{D I P-L}$ \\
\hline
\end{tabular}

TABLE V

INTRA-FINGER CONSTRAINTS FOR PRISMATIC GRASPING

\section{EVALUATION OF SIMPLIFIED HAND POSTURE DESCRIPTIONS}

The high number of DoFs (usually more than 20 DoFs) for defining the hand posture requires a longer computational time for applications that have to process this information; however, real time applications, such as gesture recognition, dynamics calculations, check collisions, etc., requires simplified models in order to obtain data faster. It is thus necessary to reduce the number of elements that make up the original hand description to fewer elements. This section describes two different simplified hand descriptions. The uncertainty of postures is not relevant for some kind of applications that can be use simplified hand descriptions in order to apply their corresponding calculations faster. These hand models are obtained by eliminating the less relevant degree of freedoms from the 24 DoF models. The most relevant DoFs have been selected according to the tables defined in section four. The two reduced versions are as follows: - Simplified hand description from 9 degrees of freedom. The DoF are obtained by selecting all independent joints. This representation includes most of the thumb, index and little finger joints. The middle and ring finger are considered coupled to the index and little finger respectively. Therefore, the selected DoF are: $\theta_{T M C(a b / a d)-T}, \theta_{I P-T}, \theta_{M C P(a b / a d)-I}$, $\theta_{D I P-I}, \theta_{D I P-M}, \theta_{D I P-R}, \theta_{C M C-L}, \theta_{M C P(a b / a d)-L}, \theta_{D I P-L}$. Simplified hand description from 6 degrees of freedom. This is a simplification of the above vector where CMC is considered only for the little finger; abduction/adduction for thumb and index; $T M C_{f e}$ and $M C P_{f e}$ for thumb and index respectively. The middle finger is totally coupled to index and ring as an interpolation of middle and little fingers. Therefore, the selected DoF are: $\theta_{T M C(a b / a d)-T}, \theta_{I P-T}$,
$\theta_{M C P(a b / a d)-I}, \theta_{D I P-I}, \theta_{C M C-L}$ and $\theta_{M C P(a b / a d)-L}$.

\section{A. Position errors of the simplified hand models}

This section shows some experiments conducted in order to evaluate both simplified hand descriptions. These experiments have been carried out for circular and precision postures. Each hand description is used for reconstructing the corresponding posture (circular and prismatic). Figures 4(a) and 4(b) show the respective postures reconstructing applied with two descriptors for circular and prismatic grasping. Table VI contains error indexes of each hand description. These error indexes were obtained measuring the error between the fingertip positions of the posture reconstructed with respect to original model of $24 \mathrm{DoF}$. Cyberglove and hand model were used to obtain the original posture that correspond to the gesture to reconstructing. It is important to indicate that these results depend on the user calibration. Data can vary $1-2 \%$ among users according to their hand size. The optimum number of DoFs for describing hand postures depends on the precision/simplification relation of each application. Therefore it is required to achieve a balance between the maximum acceptable error and minimum hand DoFs to process. According to results shown in table VI, applications that admit errors close to $10 \%$ can use $6 \mathrm{DoF}$ hand models, more accurate precisions between 5-10\% errors require 9 DoFs for hand descriptions. Finally, if positions errors must to be less than $5 \%$ then models with more than 20 DoFs have to be used.

\section{Conclusions}

Three human hand models have been analyzed in this work. The first has 24 DoFs and is based on human skeleton. This model is appropiated for very realistic manipulations. 

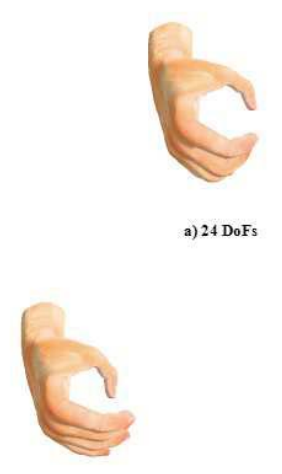

b) 9 DoFs

c) 6 DoFs

(a) Circular grasping reconstruction. a)Original Gesture b)Hand model reconstructed by 9 elements c)Hand Model reconstructed by 6 elements
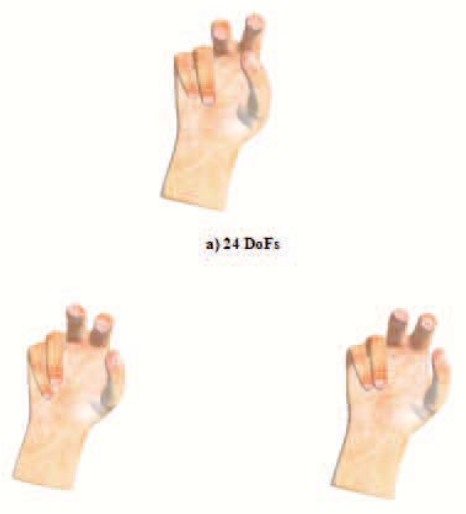

b) 9 DoFs

c) $6 \mathrm{D}_{\mathrm{oFs}}$

(b) Prismatic grasping reconstruction. a)Original Gesture b)Hand model reconstructed by 9 elements c)Hand Model reconstructed by 6 elements

Fig. 4. Grasping reconstruction

\begin{tabular}{|c|c|c|}
\hline & $\begin{array}{c}\text { Error circular } \\
\text { grasping }\end{array}$ & $\begin{array}{c}\text { Error prismatic } \\
\text { grasping }\end{array}$ \\
\hline Original model (24 DoF) & - & - \\
\hline 9 DoF hand description & $7.67 \%$ & $5.90 \%$ \\
\hline 6 DoF hand description & $13.14 \%$ & $9.18 \%$ \\
\hline
\end{tabular}

TABLE VI

ERROR INDIXES AMONG ORIGINAL HAND MODEL AND HAND POSTURE RECONSTRUCTED
This model has also been used to obtain new inter-finger and intra-finger constraints. These constraints permit allow obtaining another simplified hand descriptions. Two simplified hand descriptions with 9 and 6 DoFs have been proposed and evaluated. Finger constraints are used to estimate the rest of finger joints up to the original 24 components. These models represent a significant reduction in the number components used to describe the hand gesture. Position errors have been evaluated for both simplified descriptions. Results show that errors are close to $6 \%$ for $9 \mathrm{DoF}$ hand description and $13 \%$ for $6 \mathrm{DoF}$.

\section{ACKNOWLEDGMENTS}

This work is supported by the European commission under the IMMERSENCE integrated project of the sixth frame program (IST-4-027141-IP).

\section{REFERENCES}

[1] M. Bray, E. Koller-Meier, P. Müller, N.N. Schraudolph and L. Van Gool. Stochastic optimization for high-dimensional tracking in dense range maps. In Proc. IEEE. Vis. Image Signal Process. 501-512 (August 2005).

[2] J. J. Kuch and T. S. Huang. Human Computer Interaction via the Human Hand: A Hand Model. Signals, systems and Computers, Conference Record of the Twenty Eighth Asilomar Conference. 12521256 (1994).

[3] J. Chalfoun, M. Renault, R. Younes, F.B. Ouezdou. Muscle Forces prediction of the Human Hand and Forearm system in Highly Realistic Simulation. In Proc. IEEE/RSJ International Conference on Intelligent Robots and Systems.1293-1298 (2004)

[4] M. Renault, F.B. Oezdou. Dynamic simulation of hand-forearm system. IEEE International Workshop on Robot and Human Interactive Communication. 20-25 (2001).

[5] H. Du and E. Charbon. 3d Hand Model Fitting for Virtual Keyboard System. IEEE workshop on Applications of Computer Vision. 31-36 (February 2007).

[6] http://www.immersion.com

[7] J. Denavit and R.S. Hartenberg. A Kinematic Notation for Lower-Pair Mechanisms Based on Matrices, Journal of Applied Mechanics, vol. 77. 215 - 221 (1955).

[8] J. M Selig. Geometric fundamentals of robotics. Monographs in computer science. Springer. 85-112 (2005).

[9] W. A. Wolovich and H. Elliot, A computational technique for inverse kinematics. In Proc. 23rd IEEE Conference on Decision and Control. 1359-1363 (1984).

[10] A. Balestrino, G. De Maria, and L. Sciavicco. Robust Control of robotic manipulators, In Proceedings of the 9th IFAC World Congress. $2435-2440$ (1984).

[11] A. Kapandji. The Physiology of the Joints, volume 1. E\&S Livingstone, Edinburgh and London, 2 editions. (1970).

[12] A. Pérez Casas and M.E. Bengoechea, Functional anatomy of the apparatus locomotive, Paz Montalvo. (1978). (In Spanish).

[13] M.R. Cutkosky and P.K. Wright. Modeling Manufacturing grips and correlations with design of robotic hands. Procedings of the IEEE International Conference on Robotics and Automation. 1533-1539 (1986).

[14] Virtual Technologies. Reference Manual. CG081998-2-1 (1998).

[15] J. Lin, Y. Wu, and T.S. Huang, "Modeling the Constrains of Human Hand Motion", IEEE Human Motion Proceedings. 121-126 (2000).

[16] S. Cobos, M. Ferre M.A. Sanchéz-Urán and J. Ortego. "Constraints for Realistic Hand Manipulation". PRESENCE 2007. pp:369-370. The 10th Annual International Workshop on Presence, October 25 - 27 Barcelona, Spain (2007).

[17] C.S Fahn and H. Sun. Development of a Data Glove with Reducing Sensors Based on Magnetic Induction.IEEE Transactions on Industrial Electronics, vol. 52, No.2 (2005) 